\title{
Isomerism in 1D Coordination Polymers of Cu(II) Complexes of $N$-(2-hydroxybenzyl)-L-valine: Influence of Solvent and Coordination Sphere on the Conformation
}

\author{
Yang Xiandong, John D. Ranford, and Jagadese J. Vittal* \\ Department of Chemistry, National University of Singapore, Singapore 117543
}

Supporting Information

Infrared Spectra Typical vibrations have been assigned in the light of X-ray crystal structures. Some of the selected key IR bands of reduced Schiff base ligands have been assigned to $v(\mathrm{NH})(\mathrm{ca}$. 3194, $\left.2967 \mathrm{~cm}^{-1}\right), v_{\mathrm{as}}\left(\mathrm{CO}_{2}^{-}\right)\left(1640,1601 \mathrm{~cm}^{-1}\right), v_{\mathrm{s}}\left(\mathrm{CO}_{2}^{-}\right)\left(1400 \mathrm{~cm}^{-1}\right)$ and $v(\mathrm{CO})\left(1270 \mathrm{~cm}^{-1}\right)$ based on the assignment of analogous $\mathrm{H}_{2}$ Sgly and $\mathrm{H}_{2}$ Sala. The $v(\mathrm{OH})$ band attributed to water is not observed for all the ligands.

The X-ray crystal structures of complexes showed that the metal atoms bonded to the ligands through phenolato, amine and carboxylato groups. The $v(\mathrm{NH}), v_{\mathrm{as}}\left(\mathrm{CO}_{2}{ }^{-}\right), v_{\mathrm{s}}\left(\mathrm{CO}_{2}{ }^{-}\right)$bands shifted upon complexation, showing that the coordination involves those groups. Compared to the ligands, the bands of $v(\mathrm{NH})$ in general red have shifted $30 \mathrm{~cm}^{-1}$ to $100 \mathrm{~cm}^{-1}$ upon complexation. However, the $v_{\mathrm{s}}\left(\mathrm{CO}_{2}^{-}\right)$is moved to high energy and the trend in $v_{\mathrm{as}}\left(\mathrm{CO}_{2}^{-}\right)$is not consistent for all complexes. 
Table S1 Selected IR Absorption Bands $\left(\mathrm{cm}^{-1}\right)$ for ligands and complexes 1-4.

\begin{tabular}{|c|c|c|c|c|c|c|}
\hline Code & $v(\mathrm{OH})$ & $v(\mathrm{NH})$ & $v_{\mathrm{as}}\left(\mathrm{CO}_{2}^{-}\right)$ & $v_{\mathrm{s}}\left(\mathrm{CO}_{2}^{-}\right)$ & $\Delta^{\mathrm{b}}$ & $\begin{array}{l}\mathrm{v}(\mathrm{CO}) \\
\text { (phenolic) }\end{array}$ \\
\hline \multirow[t]{2}{*}{$\overline{\mathrm{H}_{2} \mathrm{Sval}}$} & - & 3194 (s, sp) & 1640 (vs, sp) & $1400(\mathrm{~s}, \mathrm{sp})$ & 240 & 1270 (vs, sp) \\
\hline & & 2967 (m, sp) & 1601 (vs, sp) & & 201 & \\
\hline $\mathrm{H}_{2} \mathrm{Strp}$ & - & 3111 (m, sp) & 1604 (vs, sp) & 1370 (s, sp) & & 1275 (vs, sp) \\
\hline $\mathrm{H}_{2}$ Sile & - & 2953 (m, sp) & 1595 (vs, sp) & 1398 (s, sp) & 197 & 1275 (vs, sp) \\
\hline \multirow[t]{2}{*}{$\mathrm{H}_{2}$ Sleu } & - & 3214 (m, sp) & 1639 (vs, sp) & 1389 (s, sp) & & 1266 (vs, sp) \\
\hline & & 2969 (m, sp) & & & & \\
\hline \multirow[t]{2}{*}{$\mathrm{H}_{2}$ Sphe } & - & 3193 (m, sp) & 1597 (vs, sp) & 1395 (s, sp) & & 1272 (vs, sp) \\
\hline & & 3034 (m, sp) & & & & \\
\hline \multirow[t]{2}{*}{1} & 3446 (m, br) & 3286 (m, sp) & 1638 (vs, sp) & 1390(s, sp) & 248 & 1266 (vs, sp) \\
\hline & & & 1602 (vs, sp) & 1370(s, sp) & 232 & \\
\hline \multirow[t]{2}{*}{2} & 3424 (m, br) & 3240 (s, sp) & 1643 (vs, sp) & 1395(s,sp) & 248 & 1272 (s sp) \\
\hline & 3381(m, br) & 2961 (m, sp) & 1601 (vs, sp) & 1374(s,sp) & 227 & \\
\hline 3 & 3427 (m, br) & 3276 (m, sp) & 1626 (vs, sp) & 1372(s, sp) & 254 & 1272 (vs, sp) \\
\hline \multirow[t]{2}{*}{4} & & 3293 (m, sp) & 1655 (vs,sp) & 1397(s, sp) & 258 & 1260 (vs, sp) \\
\hline & & 3202 (m, sp) & 1599 (vs,sp) & & & \\
\hline
\end{tabular}

Legend: vs = very strong, $\mathrm{s}=$ strong, $\mathrm{m}=$ medium, $\mathrm{sp}=$ sharp, $\mathrm{br}=$ broad 
Table S2. Hydrogen bond parameters for $\mathbf{1}$.

\begin{tabular}{|c|c|c|c|c|c|c|}
\hline D-H & $\mathrm{d}(\mathrm{D}-\mathrm{H})$ & $\mathrm{d}(\mathrm{H} . . \mathrm{A})$ & $<$ DHA & $\mathrm{d}(\mathrm{D} . . \mathrm{A})$ & $\mathrm{A}$ & Symmetry \\
\hline O7-H7A & 0.90 & 2.57 & 129 & $3.217(9)$ & O6 & {$[\mathrm{x}+1, \mathrm{y}, \mathrm{z}]$} \\
\hline O8-H8A & 0.90 & 1.90 & 169 & $2.783(7)$ & O6 & {$[\mathrm{x}+1, \mathrm{y}, \mathrm{z}]$} \\
\hline O8-H8B & 0.90 & 2.22 & 148 & 3.013(9) & O6 & {$[\mathrm{x}+1 / 2,-\mathrm{y}+3 / 2,-\mathrm{z}+1]$} \\
\hline O8-H8B & 0.90 & 2.54 & 151 & $3.349(7)$ & O5 & {$[\mathrm{x}+1 / 2,-\mathrm{y}+3 / 2,-\mathrm{z}+1]$} \\
\hline O9-H9A & 0.91 & 1.93 & 163 & $2.814(7)$ & $\mathrm{O} 3$ & {$[\mathrm{x}-1 / 2,-\mathrm{y}+1 / 2,-\mathrm{z}+1]$} \\
\hline О9-Н9B & 0.90 & 2.49 & 136 & 3.198(9) & O7 & {$[\mathrm{x}-1, \mathrm{y}, \mathrm{z}]$} \\
\hline O9-H9B & 0.90 & 2.58 & 112 & $3.036(7)$ & $\mathrm{O} 2$ & {$[\mathrm{x}-1, \mathrm{y}, \mathrm{z}]$} \\
\hline $\mathrm{C} 2-\mathrm{H} 2$ & 0.93 & 2.39 & 122 & 2.991(8) & O5 & \\
\hline C7-H7C & 0.97 & 2.48 & 135 & $3.239(7)$ & $\mathrm{O} 3$ & {$[\mathrm{x}-1 / 2,-\mathrm{y}+1 / 2,-\mathrm{z}+1]$} \\
\hline C12-H12 & 0.93 & 2.46 & 130 & 3.139(8) & $\mathrm{O} 2$ & \\
\hline C12-H12 & 0.93 & 2.58 & 156 & $3.450(9)$ & O9 & {$[\mathrm{x}+1, \mathrm{y}, \mathrm{z}]$} \\
\hline C17-H17A & 0.97 & 2.58 & 135 & $3.345(9)$ & O9 & \\
\hline
\end{tabular}

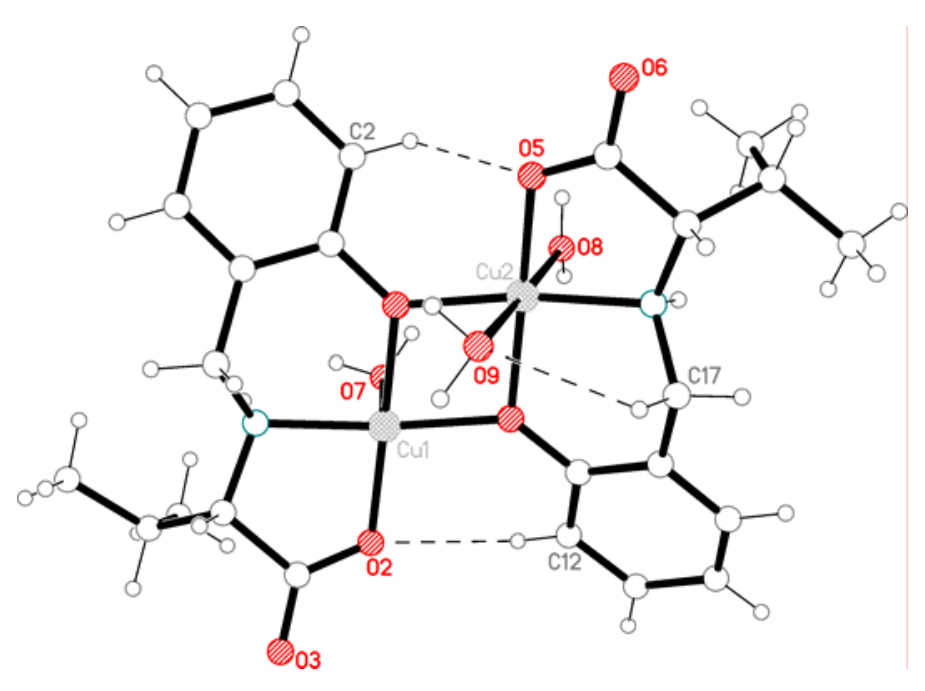

Figure S1. A view showing the intramolecular C-H $\cdots$ O bonds in $\mathbf{1}$. 


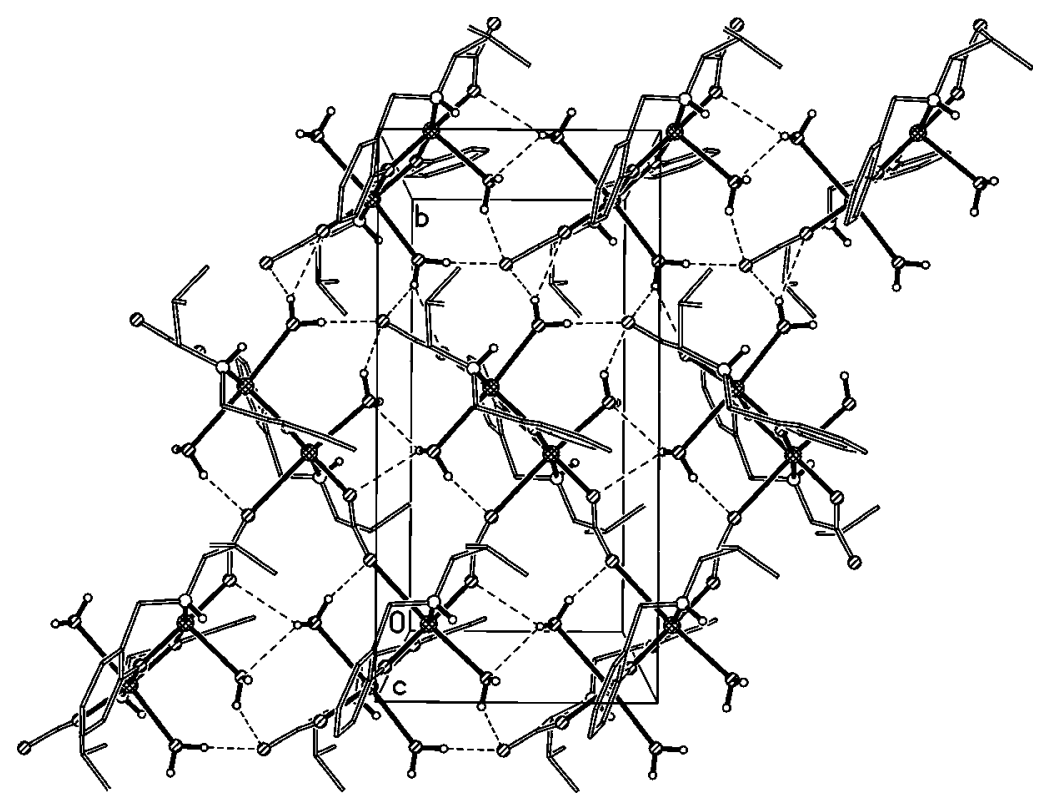

Figure S2. A view of packing structure of $\mathbf{1}$ in $a b$ plane. Only selected hydrogen atoms are shown for clarity.

Table S3. Hydrogen bonding parameters for 2.

\begin{tabular}{|c|c|c|c|c|c|}
\hline$\underline{\mathrm{D}-\mathrm{H}}$ & $\mathrm{d}(\mathrm{D}-\mathrm{H})$ & d(H..A) & $<$ DHA & d(D..A) & A Symmetry \\
\hline N2-H2 & 0.91 & 2.25 & 156 & $3.103(6)$ & O9 $[\mathrm{x}-1, \mathrm{y}-1, \mathrm{z}]$ \\
\hline O7-H7A & $0.90(3)$ & $2.04(3)$ & $173(4)$ & $2.941(5)$ & O3 $[x, y-1, z]$ \\
\hline O7-H7B & $0.90(3)$ & 1.91(3) & 172(3) & $2.801(6)$ & $\mathrm{O} 8[1-\mathrm{x}, 1 / 2-\mathrm{y}, \mathrm{z}]$ \\
\hline O8-H8B & $0.91(3)$ & $1.85(3)$ & $170(3)$ & $2.746(7)$ & O9 $[x, y-1, z]$ \\
\hline O9-H9A & $0.89(1)$ & $2.23(3)$ & 132(3) & $2.905(6)$ & $\mathrm{O} 2[1+\mathrm{x}, \mathrm{y}, \mathrm{z}]$ \\
\hline O9-H9A & $0.89(1)$ & $2.05(2)$ & $157(4)$ & $2.897(6)$ & $\mathrm{O} 3[1+\mathrm{x}, \mathrm{y}, \mathrm{z}]$ \\
\hline $\mathrm{C} 2-\mathrm{H} 2$ & 0.93 & 2.31 & 141 & $3.090(6)$ & $\mathrm{O} 5$ \\
\hline C4-H4 & 0.93 & 2.55 & 172 & $3.475(6)$ & O6 $[1-\mathrm{x}, 1 / 2+\mathrm{y}, 1-\mathrm{z}]$ \\
\hline C20-H20 & 0.98 & 2.46 & 170 & $3.429(7)$ & O6 $[-\mathrm{x}, 1 / 2+\mathrm{y}, 1-\mathrm{z}]$ \\
\hline C23-H23C & 0.96 & 2.53 & 121 & $3.142(6)$ & O6 \\
\hline C24-H24B & 0.96 & 2.53 & 122 & $3.136(7)$ & O6 \\
\hline
\end{tabular}




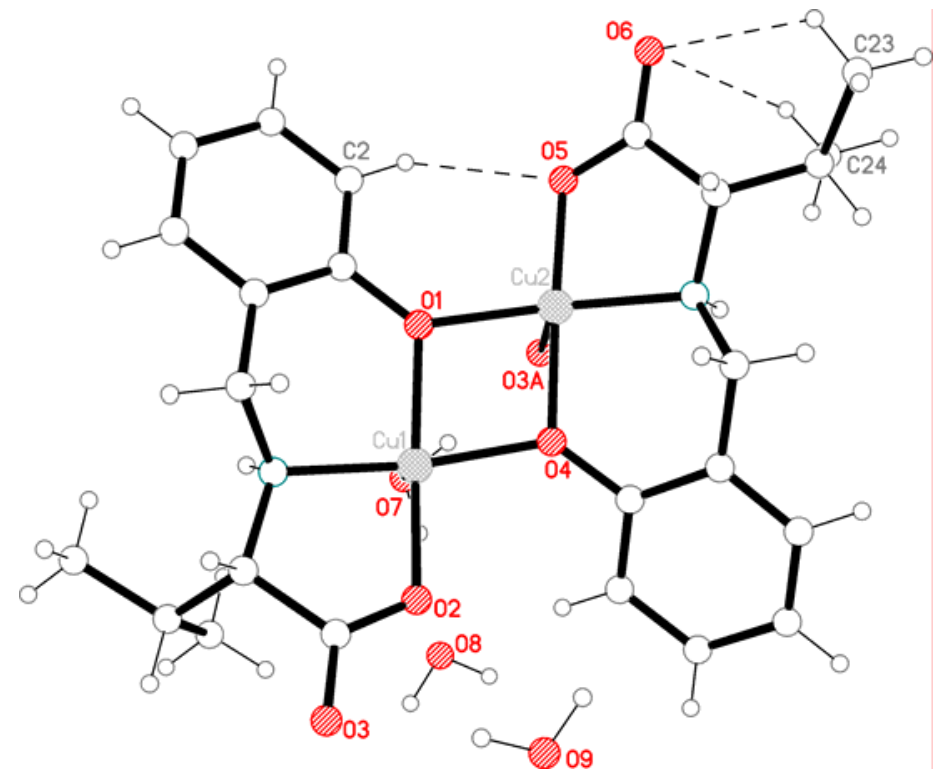

Figure S3. A view showing the intramolecular $\mathrm{C}-\mathrm{H} \cdots \mathrm{O}$ bonds in 2.

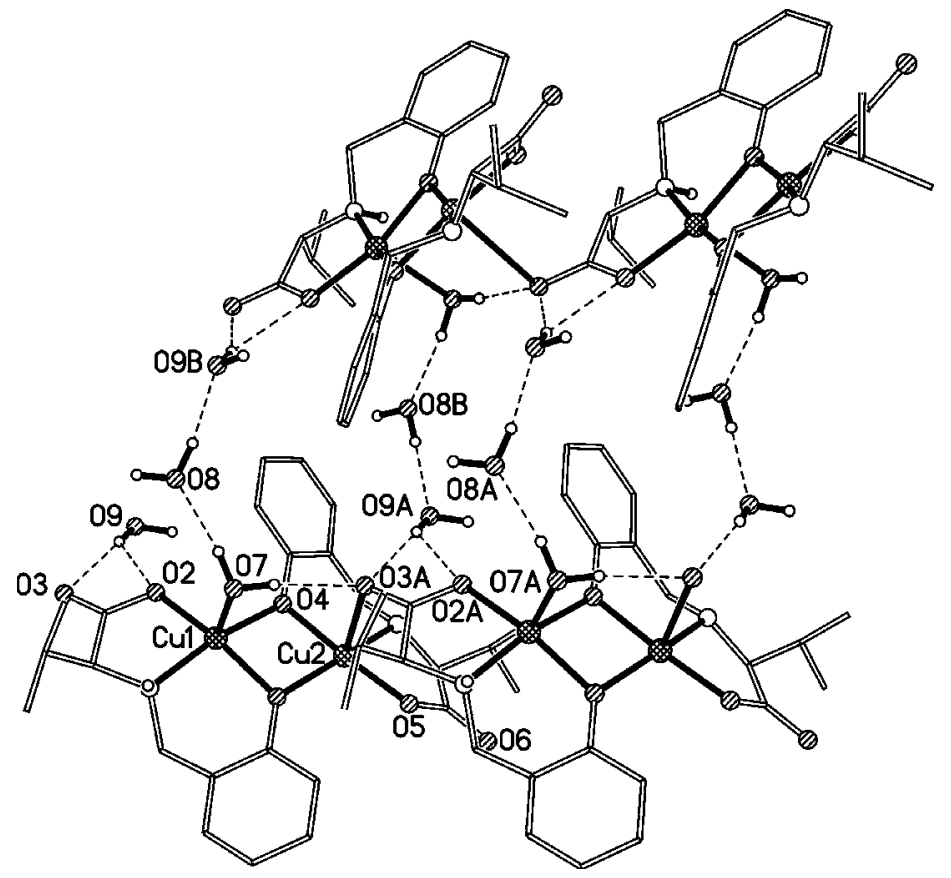

Figure S4. A view of the hydrogen bonding in the double stranded polymeric structure of 2. Only selected hydrogen atoms are shown for clarity. 
Table S4. Hydrogen bond lengths ( $\AA$ ) and angles $\left({ }^{\circ}\right)$ for 3.

\begin{tabular}{|c|c|c|c|c|c|}
\hline$\underline{\mathrm{D}-\mathrm{H}}$ & $d(D-H)$ & $\mathrm{d}(\mathrm{H} . . \mathrm{A})$ & d(H..A) & $d(D \ldots A)$ & A Symmetry \\
\hline O13-H13A & $0.89(2)$ & $1.99(2)$ & $143(2)$ & $2.760(6)$ & O9 \\
\hline O13-H13B & $0.89(3)$ & 1.93(3) & $148(2)$ & $2.724(8)$ & O1S $[-\mathrm{x}+1, \mathrm{y}-1 / 2,-\mathrm{z}]$ \\
\hline O14-H14A & $0.92(3)$ & $2.05(3)$ & $144(2)$ & $2.838(6)$ & $\mathrm{O} 3[\mathrm{x}, \mathrm{y}, \mathrm{z}+1]$ \\
\hline O14-H14B & 0.91(3) & $2.02(4)$ & $162(2)$ & 2.895(8) & O6 \\
\hline N1-H1 & 0.91 & 2.18 & 162 & $3.054(6)$ & $\mathrm{O} 12[-\mathrm{x}+1, \mathrm{y}+1 / 2,-\mathrm{z}+1]$ \\
\hline N4-H4 & 0.91 & 2.35 & 149 & $3.160(7)$ & $\mathrm{O} 2[\mathrm{x}, \mathrm{y}, \mathrm{z}+1]$ \\
\hline O1S-H1S & $0.82(2)$ & $2.21(5)$ & $121(2)$ & $2.724(9)$ & $\mathrm{O} 13[-\mathrm{x}+1, \mathrm{y}+1 / 2,-\mathrm{z}]$ \\
\hline C2-H2A & 0.93 & 2.51 & 126 & $3.137(6)$ & O5 \\
\hline C7-H7b & 0.97 & 2.56 & 128 & $3.239(7)$ & $\mathrm{O} 13$ \\
\hline C11A-H11B & 0.96 & 2.56 & 116 & $3.107(7)$ & O3 \\
\hline C11B-H11F & 0.96 & 2.32 & 125 & $2.978(9)$ & O6 \\
\hline C11C-H11H & 0.96 & 2.52 & 173 & $3.478(8)$ & O5 \\
\hline C12-H12 & 0.93 & 2.56 & 126 & $3.201(6)$ & $\mathrm{O} 2$ \\
\hline C12A-H12C & 0.96 & 2.49 & 169 & $3.433(9)$ & $\mathrm{O} 12[1-\mathrm{x}, 1 / 2+\mathrm{y}, 1-\mathrm{z}]$ \\
\hline C12D-H12K & 0.96 & 2.45 & 121 & $3.048(9)$ & $\mathrm{O} 12$ \\
\hline C18-H18 & 0.98 & 2.47 & 131 & $3.203(7)$ & $\mathrm{O} 8$ \\
\hline C22-H22 & 0.93 & 2.37 & 143 & $3.155(6)$ & $\mathrm{O} 11$ \\
\hline C27-H27A & 0.97 & 2.60 & 141 & 3.403(9) & O2s $[1-x, y-1 / 2,1-z]$ \\
\hline С30-Н30 & 0.98 & 2.54 & 101 & $2.889(7)$ & O9 \\
\hline С32-H32 & 0.93 & 2.49 & 129 & $3.159(6)$ & O8 \\
\hline
\end{tabular}




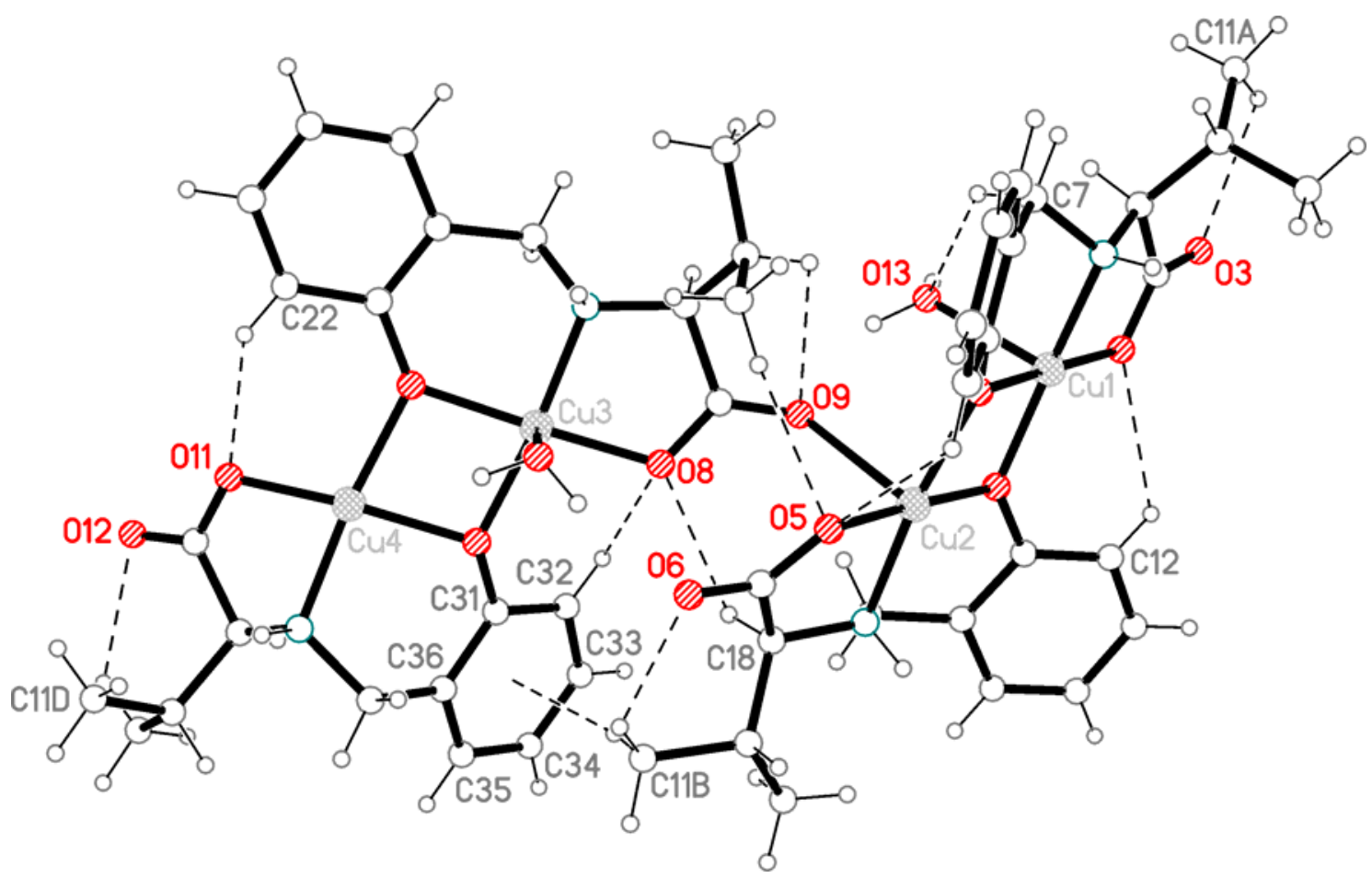

Figure S5. A view showing the intramolecular $\mathrm{C}-\mathrm{H} \cdots \mathrm{O}$ and $\mathrm{C}-\mathrm{H} \cdots \pi$ bonds in 3.

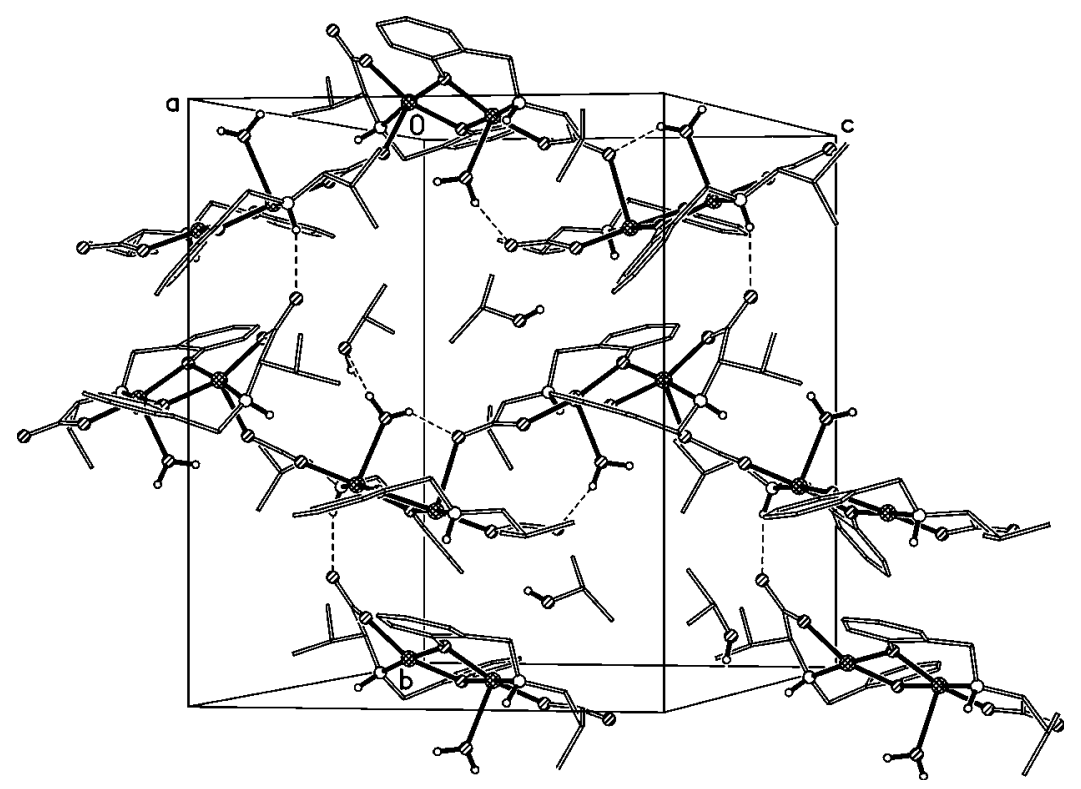

Figure S6. A portion of the structure showing the hydrogen-bonded 1D coordination polymeric structure in 3. Only selected hydrogen atoms are shown for clarity. 
Table S5. Hydrogen bond lengths $(\AA)$ and angles $\left({ }^{\circ}\right)$ for 4.

$\begin{array}{llllll}\text { D-H } & \text { d(D-H) } & \text { d(H..A) } & <\text { DHA } & \text { d(D..A) } & \text { A }\end{array}$

\begin{tabular}{|c|c|c|c|c|c|c|}
\hline N1-H1 & 0.91 & 2.23 & 163 & $3.112(7)$ & $\mathrm{O} 12$ & {$[-x+2, y+1 / 2,-z+1]$} \\
\hline N4-H4 & 0.91 & 2.33 & 145 & $3.121(8)$ & $\mathrm{O} 2$ & \\
\hline O13-H13В & 0.91 & 2.09 & 135 & $2.810(9)$ & O11 & {$[-\mathrm{x}+2, \mathrm{y}+1 / 2,-\mathrm{z}+1]$} \\
\hline O13-H13B & 0.91 & 2.24 & 148 & $3.054(9)$ & $\mathrm{O} 12$ & {$[-x+2, y+1 / 2,-z+1]$} \\
\hline O14-H14A & 0.90 & 1.96 & 171 & $2.858(7)$ & O3 & \\
\hline O14-H14B & 0.90 & 2.10 & 141 & $2.848(8)$ & O6 & {$[\mathrm{x}, \mathrm{y}, \mathrm{z}-1]$} \\
\hline O1S-H1S & 0.82 & 2.01 & 149 & $2.740(9)$ & O9 & {$[\mathrm{x}, \mathrm{y}, \mathrm{z}+1]$} \\
\hline O2SA-H2S1 & 0.82 & 2.42 & 165 & $3.22(2)$ & N3 & {$[-\mathrm{x}+2, \mathrm{y}+1 / 2,-\mathrm{z}]$} \\
\hline C2-H2A & 0.93 & 2.48 & 126 & $3.122(7)$ & $\mathrm{O} 5$ & \\
\hline C7-H7B & 0.97 & 2.50 & 129 & $3.204(7)$ & O1s & \\
\hline C12-H12 & 0.93 & 2.51 & 129 & $3.177(7)$ & $\mathrm{O} 2$ & \\
\hline C11B-H11D & 0.96 & 2.34 & 126 & 2.997(9) & O6 & \\
\hline С30-Н30 & 0.98 & 2.55 & 103 & $2.924(9)$ & O9 & \\
\hline C32-H32 & 0.93 & 2.55 & 129 & $3.213(7)$ & $\mathrm{O} 8$ & \\
\hline C12A-H12C & 0.96 & 2.39 & 164 & $3.32(1)$ & $\mathrm{O} 12$ & {$[2-\mathrm{x}, \mathrm{y}+1 / 2,1-\mathrm{z}]$} \\
\hline C12D-H12K & 0.96 & 2.38 & 124 & $3.03(1)$ & $\mathrm{O} 12$ & \\
\hline C18-H18 & 0.98 & 2.52 & 127 & $3.202(8)$ & O8 & {$[\mathrm{x}, \mathrm{y}, 1+\mathrm{z}]$} \\
\hline C27-H27A & 0.97 & 2.33 & 138 & $3.12(2)$ & O2Sa & {$[2-x, y+1 / 2,-z]$} \\
\hline C11C-H11H & 0.96 & 2.51 & 160 & $3.431(9)$ & $\mathrm{O} 5$ & {$[\mathrm{x}, \mathrm{y}, 1-\mathrm{z}]$} \\
\hline
\end{tabular}




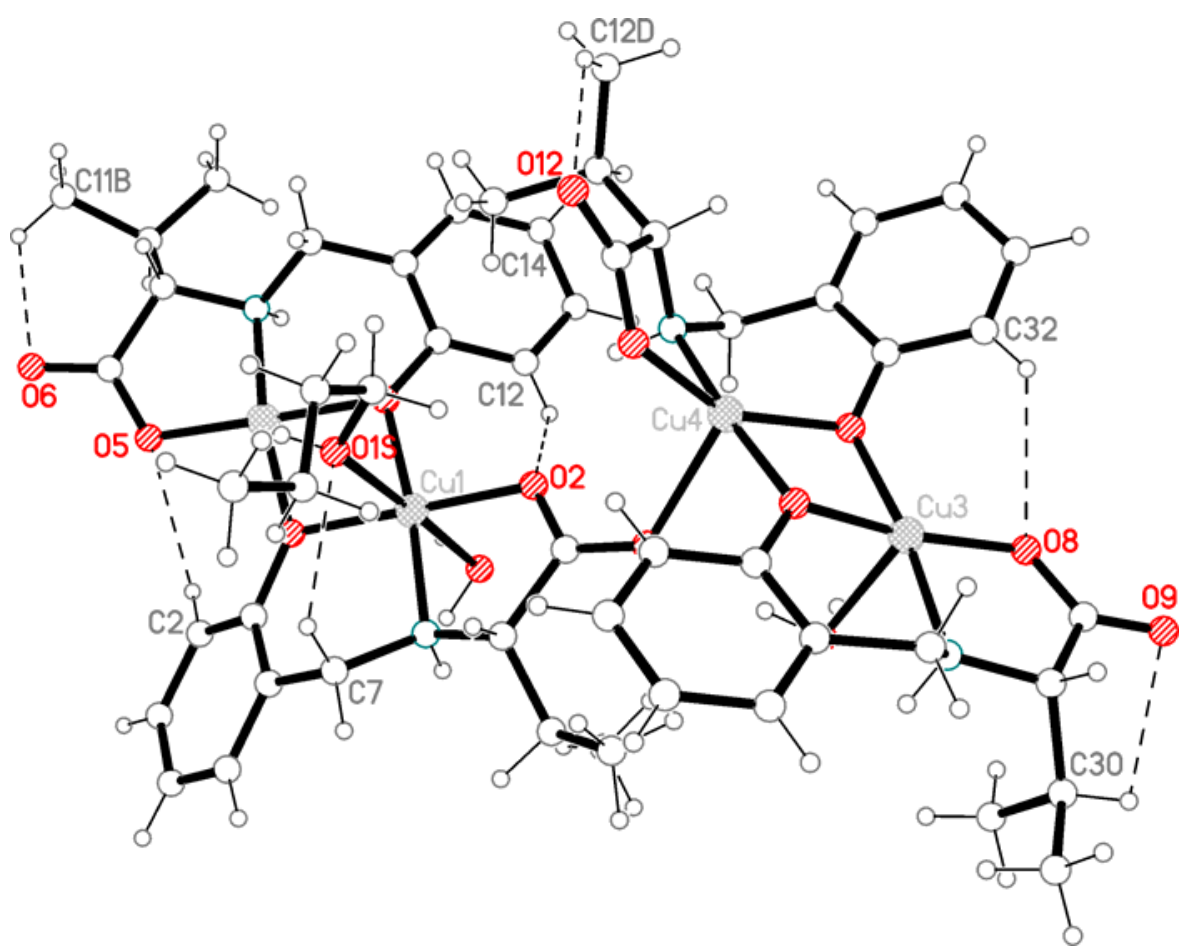

Figure S7. A view showing the intramolecular $\mathrm{C}-\mathrm{H} \cdots \mathrm{O}$ bonds in 4.

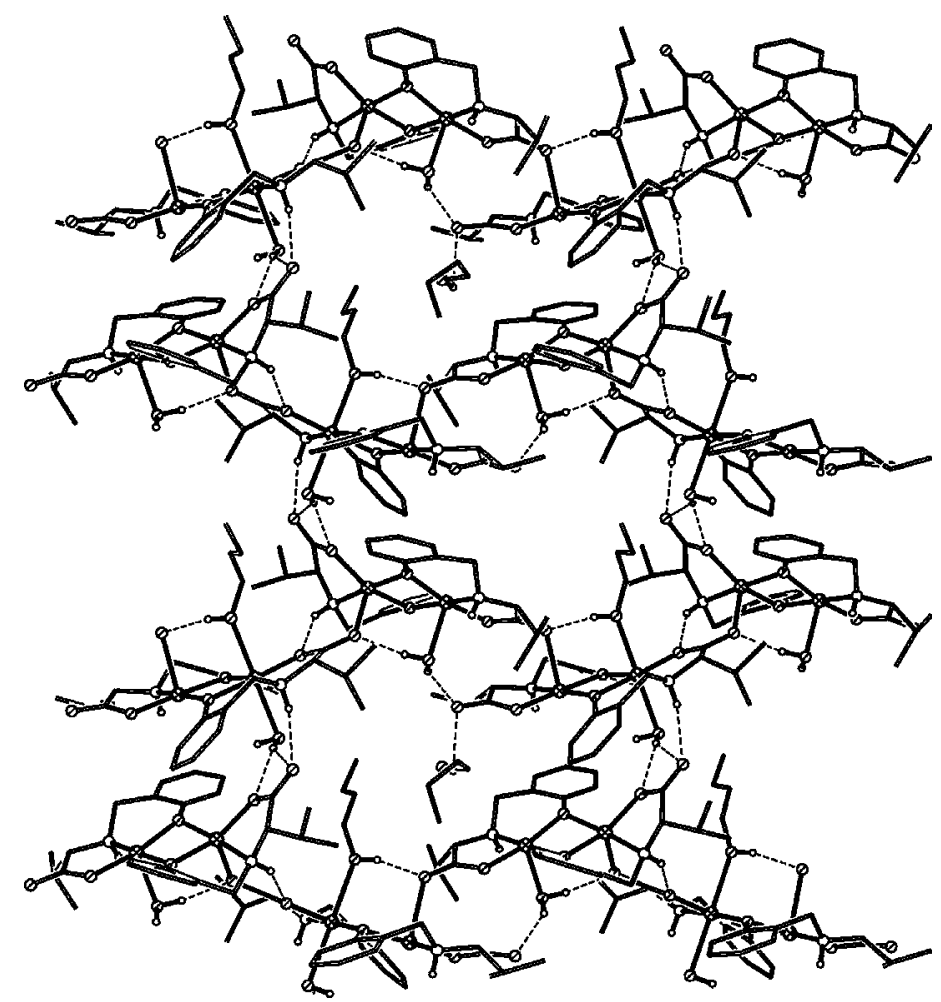

Figure S8. A section of 2D sheet in 4. Only selected hydrogen atoms are shown for clarity. 